\title{
Continuous performance task in ADHD: Is reaction time variability a key measure?
}

This article was published in the following Dove Press journal:

Neuropsychiatric Disease and Treatment

\author{
Florence Levy' \\ Andrew Pipingas ${ }^{2}$ \\ Elizabeth $\vee$ Harris $^{2}$ \\ Maree Farrow ${ }^{3}$ \\ Richard B Silberstein ${ }^{2}$ \\ 'School of Psychiatry, University of \\ New South Wales and Head, Child and \\ Family East, Prince of Wales Hospital, \\ Sydney, NSW, Australia; ${ }^{2}$ Centre \\ for Human Psychopharmacology, \\ Swinburne University of Technology, \\ Hawthorn, Victoria, Australia; ${ }^{3}$ Wicking \\ Dementia Research and Education \\ Centre, University of Tasmania, \\ Hobart, Tasmania, Australia
}

Objective: To compare the use of the Continuous Performance Task (CPT) reaction time variability (intraindividual variability or standard deviation of reaction time), as a measure of vigilance in attention-deficit hyperactivity disorder (ADHD), and stimulant medication response, utilizing a simple CPT X-task vs an A-X-task.

Method: Comparative analyses of two separate X-task vs A-X-task data sets, and subgroup analyses of performance on and off medication were conducted.

Results: The CPT X-task reaction time variability had a direct relationship to ADHD clinician severity ratings, unlike the CPT A-X-task. Variability in X-task performance was reduced by medication compared with the children's unmedicated performance, but this effect did not reach significance. When the coefficient of variation was applied, severity measures and medication response were significant for the X-task, but not for the A-X-task.

Conclusion: The CPT-X-task is a useful clinical screening test for ADHD and medication response. In particular, reaction time variability is related to default mode interference. The A-X-task is less useful in this regard.

Keywords: ADHD, continuous performance task, reaction time variability, stimulant medication

\section{Introduction}

\section{Measurement of attention deficits in ADHD}

The clinical use of the Continuous Performance Task (CPT) (Rosvold et al) ${ }^{1}$ has been characterized by a variety of CPT paradigms described in the literature (eg, Corkum and Siegel; Levy and Hobbes; Test of Variables of Attention) ${ }^{2-4}$ and the A-X-task which requires a response for an X preceded by an A. The Conners' CPT (Standard) ${ }^{5}$ requires respondents to respond to any letter except the letter X. Despite the availability of commercially marketed CPT tests, there has been no consensus on the applicability and recommended use of the CPT as a screening measure of attention (vigilance) or measurement of medication response.

\section{Inhibition vs sustained attention}

Huang-Pollock et $\mathrm{al}^{6}$ in their meta-analysis of CPT performance, evaluating vigilance deficits in attention-deficit hyperactivity disorder (ADHD), drew attention to task parameter variations having an important influence on measured or obtained outcome. For example, a traditional CPT requires subjects to make a key press to a rare signal, while inhibitory control paradigms require participants to make frequent key presses to establish a prepotent motor response, which must then be inhibited on command. The authors pointed out that because of the frequency with which a response is
Correspondence: Florence Levy Head, Child and Family East, Prince of Wales Hospital, Corner of Barker and Avoca Streets, Sydney, NSW 203I, Australia

Tel +6I 293828213

Email f.levy@unsw.ed.au 
required, and the requirement to inhibit that response, tasks such as the Conners' CPT and the Sustained Attention to Response Task ${ }^{7}$ are best described as inhibitory. It should be pointed out that compared with a more straightforward CPT X-task, the degree to which CPT A-X-task requires inhibitory activity during the A-X interval is not known, but it is hypothesized that the "A" acts as a cue to a positive response to the "X."

\section{Reaction time variability}

Increasing attention is being paid to the concept of lapses in attention, particularly during slow reaction times, as well as reaction time variability (RTV). A number of hypotheses have been proposed, including

a temporal processing deficit, a deficit in the ability to appropriately modulate very low frequency fluctuations in neural activity, inefficiency in deployment of attention by executive control processes, deficit of sustained attention, and difficulties with energetic state. ${ }^{8}$

A comprehensive meta-analysis of 319 studies of RTV in children, adolescents, and adults relative to typically developing groups investigated the context in which children and adults with ADHD differed from controls in RTV and whether between group differences were greater for RTV than for more traditional measures such as mean reaction time. ${ }^{9}$ According to the authors,

a key test of RTV's role as an ADHD core deficit will be the extent to which pharmacological and psychosocial treatments known to improve ADHD behavioral symptoms also decrease reaction time (RT) variability.

A crucial distinction in measuring RTV is the difference between individual variability in reaction time, which may be influenced by developmental stage, attentional capacity, and task variables vs group reaction time means, which are likely to reflect the inherent skew manifested in ADHD subjects' CPT performance. In this regard, the coefficient of variation $(\mathrm{CV}),(\mathrm{SD} / \mathrm{mean} \mathrm{RT})$ is designed to reflect the individual respondent's RTV controlled for his/her mean RT. Wagenmakers and Brown ${ }^{10}$ have shown that because SD in RT studies generally increases linearly with the mean, CV controls for differences in baseline speed of processing.

\section{Default mode network}

A recent explanation of RTV relates to the default mode network (DMN), which is believed to be active when individuals are engaged in introspection rather than external functions. A developing literature relating inadequate control of the DMN to ADHD has been described. ${ }^{11-13}$ Sonuga-Barke and Castellanos ${ }^{11}$ proposed that inattention in ADHD might be due to inadequate suppression of DMN activity, and thus associated with slower and more variable responses. Silberstein et al ${ }^{12-14}$ have utilized a technique termed steadystate visual evoked potential event-related partial coherence to measure the degree to which phase differences between electrode pairs remain stable across trials after the common contribution from the steady-state visual evoked potential stimulus has been removed during an A-X CPT vs a control task in ADHD vs non-ADHD children. The investigators were able to show that DMN activity was enhanced in the group of boys diagnosed with ADHD, suggesting the possibility of increased DMN activity in the CPT A-X interval in ADHD. A second study was able to demonstrate that methylphenidate suppressed the functional connectivity observed in ADHD subjects during the CPT A-X interval, while a third study demonstrated that a first dose methylphenidate induced changes in brain functional connectivity that were associated with 3 month measures of hyperactivity and inattention. ${ }^{14}$ Functional connectivity studies have thus drawn attention to the importance of DMN activity and lack of suppression during CPT performance in ADHD.

A further approach to measurement of DMN effects on executive function was reported by Yordanova et al, ${ }^{15}$ who investigated measured interference and errors during a visual Flanker task in 47 ADHD children and adults and 45 healthy controls. Analysis of error distribution across experimental blocks was carried out by fast Fourier transformation. The results demonstrated that while patients and controls exhibited multisecond rhythmic fluctuations in error behavior, ADHD patients showed additional oscillations in error generation with periodic errors at each 20-30 seconds interval. This "unique" additional periodicity was attributed to DMN effects.

\section{Medication effects}

Uebel et $a{ }^{16}$ have utilized an actigraphic approach to objectively measure methylphenidate MPH effects in ADHD children, as a method of determining differences in motor activity during structured and nonstructured analog classroom settings. While behavior ratings failed to differentiate structured from leisure time effects, actigraphy showed reduced motor behavior in structured situations vs leisure/ play. In the present context, the ADHD children in both $\mathrm{X}$-task and A-X-task designs would generally have been tested after school hours, in a structured activity. 


\section{Present study}

The present cross-sectional analyses aimed to investigate the relationship between RTV and ADHD symptomology in children performing a CPT X-task versus a CPT A-X-task. It was predicted that X-task RTV would be more closely associated with ADHD severity than A-X-task RTV and that X-task RTV would be more significantly associated with medication effects than A-X-task RTV, given that the A stimulus acts as a cue to the subsequent $\mathrm{X}$ and may mask medication effects. It was also predicted that $\mathrm{CV}$ would be a more accurate measure of severity and medication response in that $\mathrm{CV}$ controls for the RT skew found in most studies of ADHD outlined above.

\section{Method}

While Ethics approval for all studies was obtained from Ethics Committees at the relevant Hospital (Prince of Wales) and University (Swinburne University of Technology), the investigators at Swinburne University obtained individual consent forms from parents. This was not required at Prince of Wales Hospital (South Eastern Sydney Area Health Service Ethics Committee) as over 12-15 years had elapsed since testing was carried out and the study was classified as low/negligible risk and consent forms were not required.

$\mathrm{X}$-task participants were a retrospective clinical sample of 815 children (mean age $=7.9$ years; 651 male, 164 female) who were assessed and treated for possible ADHD over a previous $12-15$ year period by an experienced child psychiatrist (author-blinded). Some children were tested multiple times over several months; only the initial test was used in the analysis. The children were clinically grouped as demonstrating mild, moderate, or severe ADHD symptoms prior to being tested on the CPT X-task by the parental history and clinical observation prior to testing. Most children were tested after school.

A-X-task participants were 60 right-handed boys (as observed at testing and described by the mother) (mean age $=10.1$ years) who had been recently diagnosed with ADHD.

\section{Measures}

The children's age, gender, and handedness were recorded (Table 1). Children in the X-task condition were tested on a CPT X-task administered by computer and a response timer. A traditional CPT was used with the X-task remaining on the screen after response. The CPT task presented 80 letters containing 20 randomly distributed X's. Letters were displayed for 2 seconds with a 1.5 second interval.
Table I Participant characteristics for children in the $\mathrm{X}$-task and A-X-task cohorts

\begin{tabular}{lll}
\hline Characteristics & X-task & A-X-task \\
\hline $\begin{array}{l}\text { Age in months, } \\
\text { range (mean } \pm \text { SD) }\end{array}$ & $42-196,(94.3 \pm 26.9)$ & $88-167,(120.7 \pm 21.7)$ \\
$\begin{array}{l}\text { Gender, N (\%) } \\
\quad \text { Male }\end{array}$ & \\
$\quad$ Female & $651(79.8)$ & $60(100)$ \\
Handedness, N (\%) & $164(20.1)$ & \\
$\quad$ & & \\
$\quad$ Right & $627(77.0)$ & $60(100)$ \\
$\quad$ Left & $108(13.3)$ & - \\
$\quad$ Other & $79(9.7 \%)$ & - \\
\hline
\end{tabular}

Note: ' - ' indicates not measured.

Individual response times were recorded for each of the 20 target trials, with the mean of these (excluding the first two trials) used as the RT variable. RTV was calculated as the variance of the RT.

In order to investigate RTV in a separate sample, using a different A-X CPT paradigm, the ADHD cohort was subjected to a similar analysis. For consistency, hyperactive and inattentive parental ratings at baseline were treated as combined symptom scores and divided into low, medium, and high scores at baseline. The A-X-task presented 80 letters with 16 randomly presented targets and was repeated (160 letters with 32 targets in total). Children were required to respond to the appearance of an $\mathrm{X}$ that was preceded by an A. Letters were displayed for 2 seconds with a 1.5 second interval. RT and RTV were calculated as mentioned earlier.

\section{Statistical analysis}

For the X-task, independent samples $t$-tests were initially used to compare boys' versus girls' and left- versus righthanded children's performance on the X-task. Participants with fewer than $75 \%$ valid responses were excluded from the analysis $(\mathrm{N}=15)$. For the $\mathrm{X}$-task, $t$-tests indicated that there was no significant difference between boys and girls for RT, RTV, or CV, or between left- and right-handed children ( $p$-values $>0.05$ ). Therefore, all groups were combined for further analyses.

Analyses of covariance (ANCOVAs) were conducted to investigate RT, RTV, and CV in relation to ADHD level. RTV and CV were positively skewed, so log 10 transformations were applied. RT was nearly normally distributed and did not require transformation. For RTV, $\log$ RTV was entered as the dependent variable, ADHD severity rating was the independent variable, and age in years, sex, and RT were entered as covariates. With RT and $\log \mathrm{CV}$ as the dependent variable, ADHD severity rating was the independent variable, and age in years and sex were entered as covariates. 
For the A-X-task, RTV was extremely positively skewed, so a $\log 10$ transformation was applied. RT data were also positively skewed, and so a square root transformation was used. CV was close to normally distributed. ANCOVAs were conducted as for the $\mathrm{X}$-task data, with ADHD level rating as the independent variable in each.

Finally, a smaller subset of patients $(\mathrm{N}=25)$ had X-task data from multiple time points, with and without medication. (At the time of testing, immediate-release methylphenidate was the primary medication used to treat ADHD children at the clinic in dose ranges from 10 to $30 \mathrm{mg}$ daily after breakfast and lunch depending on size and weight). From these patients, two time points were selected, comparing one session on medication and one not on medication (school holidays) where the patient was closest in age. For the A-X-task, all participants underwent testing both with and without medication. The normality assumption was tested on the distribution of difference scores between sessions (ie, T2 - T1). These variables were normally or near-normally distributed for all variables. Paired samples $t$-tests were conducted to determine statistical differences in RT, RTV, and CV between sessions.

\section{Results}

Demographic information for the children in the X-task and A-X-task groups is provided in Table 1. Means and standard deviations of RT, RTV, and CV are provided in Tables 2 and 3. In the X-task, 410 participants had no omissions, 164 had one omission, 67 had two omissions, with the remaining having 3 or more omissions. Mean (SD) commission errors was 1.97 (4.5), although $61.5 \%$ had no commission errors. In the A-X-task, 35 participants had no omissions, ten had one omission, six had two omissions, and the remaining

Table 2 Mean RT, RTV, and CV for children with low, medium and high severity of ADHD in the X-task clinical cohort

\begin{tabular}{llll}
\hline ADHD severity & Mean & SD & N \\
\hline Mean RT (ms) & & & \\
Low ADHD & 766 & 199 & 324 \\
Med ADHD & 808 & 229 & 273 \\
High ADHD & 844 & 216 & 200 \\
RTV (ms) & & & \\
Low ADHD & 54,025 & 67,084 & 323 \\
Med ADHD & 79,630 & 91,685 & 273 \\
High ADHD & 89,100 & 83,207 & 198 \\
CV & & & \\
Low ADHD & 0.2587 & 0.1196 & 324 \\
Med ADHD & 0.2882 & 0.1097 & 274 \\
High ADHD & 0.3049 & 0.1250 & 200 \\
\hline
\end{tabular}

Abbreviations: ADHD, attention-deficit hyperactivity disorder; $\mathrm{CV}$, coefficient of variation; RT, reaction time; RTV, reaction time variability.
Table 3 Mean RT, RTV, and CV for children with low, medium, and high level of ADHD in the A-X-task cohort

\begin{tabular}{clll}
\hline ADHD level & Mean & SD & N \\
\hline Mean RT (ms) & & & \\
Low ADHD & 545 & 166 & 18 \\
Med ADHD & 604 & 116 & 20 \\
High ADHD & 560 & 143 & 19 \\
RTV (ms) & & & \\
Low ADHD & 42,988 & 46,486 & 18 \\
Med ADHD & 58,874 & 47,602 & 20 \\
High ADHD & 41,298 & 38,189 & 19 \\
CV & & & \\
Low ADHD & 0.3132 & 0.1259 & 18 \\
Med ADHD & 0.3645 & 0.1404 & 20 \\
High ADHD & 0.3323 & 0.1025 & 19 \\
\hline
\end{tabular}

Abbreviations: $\mathrm{ADHD}$, attention-deficit hyperactivity disorder; $\mathrm{CV}$, coefficient of variation; RT, reaction time; RTV, reaction time variability.

had three or more omissions. Mean (SD) commission errors was 2.1 (2.4), with $21.6 \%$ having no commission errors.

Some participants had missing data for particular analyses, including three participants with missing data for parental response. Missing data was determined to be missing at random.

\section{CPT X-task}

ANCOVA indicated that although RT increased with higher ADHD severity ratings, (Table 2), the difference was relatively small and not statistically significant after controlling for covariates $(\mathrm{F}(2,792)=2.227, p=0.109)$. Of these covariates, age was significant $(p<0.001)$ and sex was not significant ( $p=0.388)$.

RTV was significantly different between the different severity ratings, after controlling for covariates $\left(\mathrm{F}(2,788)=8.37, p<0.001\right.$, partial $\left.\eta^{2}=0.021\right)$, indicating a higher RTV in subjects with higher severity ratings (Table 2). Age was a significant covariate $(p<0.001)$ but sex was not $(p=0.238)$.

$\mathrm{CV}$ was also significantly different between the different severity ratings, after controlling for covariates $\left(\mathrm{F}(2,789)=9.20, p<0.001\right.$, partial $\left.\eta^{2}=0.023\right)$, indicating a higher CV in subjects with higher severity ratings (Table 2). Again, age was a significant covariate $(p<0.001)$ but sex was not $(p=0.248)$.

\section{CPT A-X-task}

For the A-X-task, ANCOVA indicated there was no significant difference in RT between symptom level groups after controlling for age $(\mathrm{F}(2,57)=2.32, p=0.110)$, which was a significant covariate $(p<0.001)$ (Table 3$)$. 
Table 4 Mean RT, RTV, and CV for children with and without medication in the $\mathrm{X}$-task and A-X-task cohorts

\begin{tabular}{|c|c|c|c|c|c|}
\hline \multirow[t]{2}{*}{ Tasks } & \multicolumn{2}{|c|}{ No medication } & \multicolumn{2}{|c|}{$\begin{array}{l}\text { With } \\
\text { medication }\end{array}$} & \multirow[t]{2}{*}{$\mathbf{N}$} \\
\hline & Mean & SD & Mean & SD & \\
\hline \multicolumn{6}{|l|}{ X-task } \\
\hline Mean RT (ms) & 769 & 190 & 751 & 232 & 25 \\
\hline RTV (ms) & 51,732 & 57,151 & 38,318 & 34,009 & 25 \\
\hline $\mathrm{CV}$ & 0.4874 & 0.1702 & 0.2355 & 0.0708 & 25 \\
\hline \multicolumn{6}{|l|}{ A-X-task } \\
\hline Mean RT (ms) & 566 & 142 & 570 & 142 & 60 \\
\hline RTV (ms) & 46,876 & 43,687 & 55,354 & 56,620 & 60 \\
\hline$C V$ & 0.3351 & 0.1220 & 0.3517 & 0.1570 & 60 \\
\hline
\end{tabular}

Abbreviations: $C V$, coefficient of variation; $R T$, reaction time; $R T V$, reaction time variability.

ANCOVA revealed no significant difference in RTV between low-, medium-, and high-level groups after controlling for age and RT $(\mathrm{F}(2,57)=0.510, p=0.603)$. Of the covariates, RT was significant $(p<0.001)$ but age was not ( $p=0.417)$ (Table 3).

ANCOVA revealed no significant difference in $\mathrm{CV}$ between low-, medium-, and high-level groups in the A-Xtask after controlling for age $(\mathrm{F}(2,57)=0.901, p=0.412)$ (Table 3). Age was not a significant covariate $(p=0.092)$.

\section{Medication analyses}

For the X-task analyses of medication, there was no significant difference in RT between sessions with medication or without $(\mathrm{t}(24)=0.545, p=0.591)$. RTV was lower in sessions on medication compared to no medication (Table 4), but the difference was not significant $(\mathrm{t}(24)=1.378, p=0.181)$. $\mathrm{CV}$ was lower in sessions on medication compared to no medication, and the difference was significant $(\mathrm{t}(24)=6.906, p<0.001)$.

For the A-X-task, there was no significant difference between sessions with or without medication (Table 4) for $\mathrm{RT}(\mathrm{t}(59)=0.802, p=0.426), \mathrm{RTV}(\mathrm{t}(59)=0.283, p=0.778)$ or $\mathrm{CV}(\mathrm{t}(59)=0.720, p=0.475)$.

\section{Discussion}

With the advent of the Diagnostic and Statistical Manual of Mental Disorders, 5th Edition (DSM5) ${ }^{17}$ emphasis on diagnostic traits and the Research Domain Criteria ${ }^{18}$ philosophy of utilizing objective psychometric measurement in relation to behavioral symptomatology and ultimate underlying physiology, it was thought useful to revisit objective psychometric tests that have been utilized in the measurement of ADHD.

The present review and data draws attention to the differing traits measured by differences in CPT paradigms. These appear to vary from measuring inhibition as in the "standard"
Conners' CPT which requires the respondent to press an appropriate key for any letter except the letter X. Although our tasks cannot be compared statistically, the better measure of attention (vigilance) appears to be the RTV during the simple X-task CPT as demonstrated by the present data. On the other hand, the CPT A-X-task provides an "A" cue that appears to diminish the discriminatory power of RTV, though reaction time remained significant as a covariant.

The work of Sonuga-Barke and Castellanos ${ }^{11}$ and of the Silberstein group ${ }^{12-14}$ is consistent with DMN interference as a cause of RTV but may not explain the RT skew often described in measures of ADHD, and could result from separate physiological processes. ${ }^{9}$ In this regard, the work of Yordonova et al, ${ }^{15}$ based on fast Fourier analysis of the frequency of error distribution during a visual Flanker task, suggested two parallel oscillatory patterns, one of which was common to ADHD and normal subjects $(\sim 0.08 \mathrm{~Hz})$ more apparent over time, and a superimposed $(\sim 0.05 \mathrm{~Hz})$ frequency that was only manifest in ADHD subjects throughout the course of sampled time blocks. The investigators postulated that the latter oscillations interfered with executive functions in ADHD children. This is consistent with the present CPT X-task findings of executive deficits expressed as RT variability throughout the continuous task.

The present CPT X-task appears to give an accurate predictive measure of degree of medication response in children referred for possible ADHD and could be useful as an objective screening test for underlying attention problems and medication response.

\section{Limitations}

The main limitation of the present study is that different cohorts and different clinical ratings of ADHD severity were used in the X-task (clinical rating) versus the A-Xtask (parental ratings), and thus the differences in task performance were not able to be compared statistically. However, the very strong association between RTV and ADHD severity in the X-task and the lack of any such relationship in the A-X-task supports the contention that $\mathrm{X}$-task RTV is a measure of sustained attention, whereas the A-X-task may measure inhibitory capacity, while the "A" cue may reduce RTV. The clear relation between the X-task RTV and clinical severity suggests that it may be a useful screening test for ADHD and for monitoring medication response. The optimal measure appears to be $\mathrm{CV}$, given the internal control for skew as well as better sensitivity to medication. However, the current medication findings should be replicated in a larger sample. 


\section{Disclosure}

The authors report no conflicts of interest in this work.

\section{References}

1. Rosvold HE, Mirsky AF, Sarason I, Bransome ED, Beck LH. A continuous performance test of brain damage. J Consult Psychol. 1956; 20(5):343-350

2. Corkum PV, Siegel LS. Is the continuous performance task a valuable research tool for use with children with attention-deficit-hyperactivity disorder? J Child Psychol Psychiatr. 1993;34(7):1217-1239.

3. Levy F, Hobbes G. The diagnosis of attention deficit disorder (Hyperkinesis) in children. J Am Acad Child Psychiatry. 1981;20(2):376-384.

4. Introducing the Test of Variables of Attention. Los Alamitos, CA: The TOVA Company; 1991.

5. Conners' Continuous Performance Test (CPT) [computer program]. St Edmunds, UK: Multi-Health Systems Inc; 1992.

6. Huang-Pollock CL, Karalunas SL, Tam H, Moore AN. Evaluating vigilance deficits in ADHD: a meta-analysis of CPT performance. J Abnorm Psychol. 2012;121(2):360-371.

7. Baron IS. Test of everyday attention for children. Child Neuropsychol. 2001;7(3):190-195.

8. Tamm L, Narad ME, Antonini TN, O’Brien KM, Hawk LW, Epstein JN. Reaction time variability in ADHD: a review. Neurotherapeutics. 2012; 9(3):500-508.

9. Kofler MJ, Rapport MD, Sarver DE, et al. Reaction time variability in ADHD: a meta-analytic review of 319 studies. Clin Psychol Rev. 2013; 33(6):795-811.
10. Wagenmakers EJ, Brown S. On the linear relation between the mean and the standard deviation of a response time distribution. Psychol Rev. 2007:114(3):830-841.

11. Sonuga-Barke EJS, Castellanos FX. Spontaneous attentional fluctuations in impaired states and pathological conditions: a neurobiological hypothesis. Neurosci Biobehav Rev. 2007;31(7):977-986.

12. Silberstein RB, Pipingas A, Farrow M, Levy F, Stough CK. Dopaminergic modulation of default mode network brain functional connectivity in attention deficit hyperactivity disorder. Brain Behav. 2016;6(12):e00582.

13. Silberstein RB, Pipingas A, Farrow M, Levy F, Stough CK, Camfield DA. Brain functional connectivity abnormalities in attention-deficit hyperactivity disorder. Brain Behav. 2016;6(12):e00583.

14. Silberstein RB, Levy F, Pipingas A, Farrow M. First-dose methylphenidate-induced changes in brain functional connectivity are correlated with 3-month attention-deficit/hyperactivity disorder symptom response. Biol Psychiatry. 2017;82(9):679-686.

15. Yordanova J, Albrecht B, Uebel H, et al. Independent oscillatory patterns determine performance fluctuations in children with attention deficit/ hyperactivity disorder. Brain. 2011;134(6):1740-1750.

16. Uebel H, Albrecht B, Kirov R, et al. What can actigraphy add to the concept of labschool design in clinical trials? Curr Pharm Des. 2010; 16(22):2434-2442.

17. American Psychiatric Association. Diagnostic and Statistical Manual of Mental Disorders: DSM-5. 5th ed. Washington, DC: American Psychiatric Association; 2013.

18. Insel T, Cuthbert B, Garvey M, et al. Research Domain Criteria (RDoC): toward a new classification framework for research on mental disorders. Am J Psychiatry. 2010;167(7):748-751.
Neuropsychiatric Disease and Treatment

\section{Publish your work in this journal}

Neuropsychiatric Disease and Treatment is an international, peerreviewed journal of clinical therapeutics and pharmacology focusing on concise rapid reporting of clinical or pre-clinical studies on a range of neuropsychiatric and neurological disorders. This journal is indexed on PubMed Central, the 'PsycINFO' database and CAS,
Dovepress

and is the official journal of The International Neuropsychiatric Association (INA). The manuscript management system is completely online and includes a very quick and fair peer-review system, which is all easy to use. Visit http://www.dovepress.com/testimonials.php to read real quotes from published authors. 